\title{
Electromotive Drug Administration for Treatment of Therapy- Refractory Overactive Bladder
}

\author{
A. Gauruder-Burmester, A. Biskupskie, A. Rosahl, R. Tunn
}

German Pelvic Floor Center, Urogynecology Section, St. Hedwig Hospital Berlin, Berlin, Germany

\begin{abstract}
Purpose: Evaluate the benefits of electromotive drug administration (EMDA) as an alternative technique in patients with chronic overactive bladder in terms of improvement of symptoms, quality of life, and sexuality.

Material and Methods: A total of 72 patients with therapy-refractory overactive bladder according to the ICS (International Continence Society) definition, were treated by EMDA. The regimen consisted of three treatment cycles, each with 3 instillations at 2-week intervals. The solution instilled consisted of $100 \mathrm{~mL} \mathrm{4 \%} \mathrm{lidocaine,} 100 \mathrm{~mL}$ distilled water, $40 \mathrm{mg}$ dexamethasone, and $2 \mathrm{~mL}$ epinephrine. Peri-interventionally, a urine test and close circulatory monitoring were performed. All women underwent urodynamic testing and cystoscopy and kept a voiding diary. A comprehensive history was obtained, a quality of life questionnaire administered, and a gynecologic examination performed before initiation of therapy. The women underwent follow-up at 12 months after the end of therapy.

Results: The patients had a mean age of $63( \pm 11.2)$ years. Bladder capacity improved significantly by $109 \mathrm{~mL}( \pm 55 \mathrm{~mL})$ in $51(71 \%)$ patients $(\mathrm{p}=0.021)$. The number of micturitions/day decreased significantly to $7( \pm 2)(\mathrm{p}=0.013)$. Quality of life was improved in 54 patients $(75 \%) ; p=0.024)$ and sexuality in $39(54 \%) ; p=0.020)$.

Conclusions: The results suggest that EMDA can improve both quality of life and sexuality in patients with therapy-refractory chronic overactive bladder.
\end{abstract}

Key words: overactive bladder; therapy; quality of life; sexuality

Int Braz J Urol. 2008; 34: 758-64

\section{INTRODUCTION}

Overactive bladder (OAB) has a socioeconomic impact that is comparable to that of diabetes mellitus. As life expectancy is increasing, this condition will become even more important in the future. In the new classification of the International Continence Society (ICS) (1), an overactive bladder is defined as a complex of symptoms comprising frequency, nocturia, and sudden compelling desire to void with or without urge incontinence. $\mathrm{OAB}$ has a prevalence of approximately $17 \%$. The etiology of OAB comprises neurogenic and non-neurogenic detrusor overactivity and detrusor hypersensitivity; also discussed is urothelial dysfunction (2). Most affected patients seek medical advice very late, at a time when the symptoms have progressed to a stage where permanent treatment is required. Compliance is poor in most patients who have already tried a variety of therapies that failed or led to minor improvement at best. Most approaches rely on medications. Comparison of the effects of different agents is difficult because the reported studies investigating these therapies differed in design and patients population investigated. Moreover, physiotherapeutic education and behavioral training or bladder drill are offered either alone or in combina- 
tion with medical treatment. After these therapeutic options fail to cure or improve $\mathrm{OAB}$, physicians and patients are often at a loss. The cost for incontinence aids increases and many of the patients in whom all therapeutic options have been tried are on long-term sick leave or incapable of gainful employment. We therefore intended to offer a treatment that has previously been established and has been shown to be effective in patients diagnosed with OAB. Electromotive drug administration (EMDA) combines iontophoresis and electrophoresis for targeted delivery of drugs to deep tissue layers. This is achieved by means of an electrical field created between electrodes. Passive diffusion overcomes some of the limitations of conventional drug administration and achieves a higher local concentration of the drug while reducing possible toxic effects. At the same time, EMDA can be used to improve bladder capacity by means of bladder distension without the need for anesthesia. Our aim was to establish an effective, permanent or repeatedly applicable therapy for patients with chronic OAB while at the same time minimizing adverse effects.

In the study presented here, EMDA therapy was used in 72 patients with overactive bladder.

Treatment consisted of a stepwise approach. Patients were initially treated with anticholinergics. If two anticholinergics failed, patients were treated with uropol-S ${ }^{\circledR}$ instillation. Alternatively, electrostimulation with a current of $20 \mathrm{~Hz}$ or less combined with bladder training was offered. If this also failed, patients underwent urodynamic and cystoscopic diagnostic workup. Patients in whom the diagnosis of OAB was confirmed were then treated by EMDA. The aim of our study was to show that EMDA therapy improves the patients' quality of life and sexuality.

\section{MATERIALS AND METHODS}

A total of 72 patients with therapy-refractory overactive bladder according to the definition of the ICS (3) received EMDA treatment. Of the 85 patients initially eligible for the study, $13(20 \%)$ were excluded from treatment because they had abnormal pelvic floor electromyography (EMG) findings.

Inclusion Criteria: Patients were included if they had a history of OAB persisting for over 24 months and therapeutic attempts with at least two different anticholinergic medications and at least 6 months of physiotherapy without improvement or cure of symptoms: frequency (voiding more than 8 times a day), nocturia (voiding more than once a night), and urge incontinence. The voiding frequency was determined by means of a voiding diary the patients kept for three days. All patients underwent urodynamic testing before and after treatment. The following parameters were determined: first urge to urinate (normal: one third of total bladder capacity), bladder capacity (normal: $>300 \mathrm{~mL}$ ), and positive detrusor contractions. Presence of residual urine was an exclusion criterion. Residual urine was determined by ultrasound and clinically by means of catheterization during urodynamic testing. A cystoscopy was performed in patients who had their last cystoscopy more than 6 months earlier.

A vaginal examination served to rule out pelvic organ prolapse and to evaluate the mucosa (4). Introital ultrasound was performed in a standardized manner (5) and served to verify abnormal findings (e.g., exclusion of urethral diverticula, tumors). Each patient was administered a quality of life (6) and sexual exploration questionnaire (7) before and after treatment. Quality of life was assessed using the validated Kings Health questionnaire. The questionnaire on sexuality was compiled by the Institute for Sexual Medicine of Humboldt University in Berlin and is also a validated measuring instrument.

Voiding frequency, nocturia, first urge, bladder capacity, quality of life, and sexuality were compared before and after treatment. Pad counts were not done because the results were not comparable as patients use a diversity of products.

Each patient received three treatment cycles each with three instillations at two-week intervals $( \pm 3$ days). The solution instilled consisted of $100 \mathrm{~mL} \mathrm{4 \%}$ lidocaine hydrochloride ( $\mathrm{NaCl}$-free), $100 \mathrm{~mL}$ distilled water, $40 \mathrm{mg}$ dexamethasone sodium phosphate, and $2 \mathrm{~mL}$ epinephrine. Close circulatory monitoring was performed peri-interventionally (6 times a day) and a urine test was done. Patients were hospitalized for three days.

The EMDA system used in the study consists of a control unit (Physionizer ${ }^{\circledR}$ 30, UROMED) and a catheter electrode with a diameter of $16 \mathrm{~F}$ and a 
shaft length of $40 \mathrm{~cm}$. The electrode (CE-DAS ${ }^{\circledR}$ UROGENICS ${ }^{\circledR} /$ Ag 9701) was inserted and then blocked by means of a balloon inflated with $3 \mathrm{~mL}$ of air. The bladder was rinsed with distilled, sterile water and drained. Bladder tolerance was then determined as the level at which symptoms occurred. The medications instilled had a total volume that was $5-10 \mathrm{~mL}$ below the bladder capacity at which the individual patient experienced symptoms. Two patch electrodes were applied to the abdomen and the Physionizer ${ }^{\circledR}$ was connected to the catheter and the patch electrodes. Patients were treated for 20-25 min with application of 15-25 mA. Current was increased at a rate of 30-60 uA/sec. The frequency was $2.5 \mathrm{kHz}$. The current applied was direct current with rectangular impulses. Absolute contraindications were urinary tract infection before therapy, allergy/ intolerance of local anesthetics, massive intravesical bleeding, pregnancy, patients taking medications of the group of monoaminoxidase inhibitors and cardiac pacemaker.

Patients underwent follow-up 12 months after the last treatment.

Statistical analysis was performed by the Institute of Dr. Matthias Koch-Moeck using MedCalc 9.0 .

\section{RESULTS}

\section{History and Diseases}

The patients had a mean age of $63( \pm 11.2)$ years. With a Body Mass Index of $31( \pm 4.1)$, the majority of patients were overweight. Overweight was found to correlate with the symptoms of OAB.

Fifteen patients $(21 \%)$ had a history of antiincontinence surgery and 19 (27\%) had undergone prolapse surgery. There was no correlation with OAB. Five patients were nulliparae. The number of deliveries in the other patients ranged from 1 to 7 . The number of deliveries had no effect on the severity of symptoms. Eighteen patients with type II diabetes mellitus $(25 \%)$ had no EMG changes of the pelvic floor. Twelve patients (17\%) had neurological disorders that did not correlate with their urogynecologic diseases (Figures-1 and 2).

Eighteen patients $(25 \%)$ were previously treated by electrostimulation, $46(64 \%)$ by a combination of electrostimulation and biofeedback (Table$1)$.

\section{Clinical Examination}

Pelvic floor muscle contraction was assessed using the Oxford grading system (8). The average strength was $2( \pm 2)$ and an association was found between poor strength and premature first urge $(\mathrm{p}=$ 0.0003).

The gynecologic examination did not show any prolapse in any of the patients (ICS stage 0 ). Fiftyone $(71 \%)$ patients had a $\mathrm{pH}$ of $4.2( \pm 0.2)$, indicating good estrogenization, while $21(29 \%)$ had poor estrogenization with a $\mathrm{pH}$ of $5.5( \pm 0.1)$. The patients with poor estrogenization were prescribed ovestin ovula pessaries (for vaginal application every 3 days for 3 months). Introital ultrasound did not show any significant pathology.

Table 1 -Medications before start of electromotive drug administration (EMDA) treatment.

\begin{tabular}{lc}
\hline Medication Before Start of EMDA Treatment & Number / Percentage \\
\hline Trospium chloride & $32 / 44 \%$ \\
Tolterodine & $34 / 47 \%$ \\
Oxybutynin & $19 / 26 \%$ \\
Oxybutynin transdermal patches & $15 / 21 \%$ \\
Propiverine & $19 / 14 \%$ \\
Sodium chondroitin sulfate (uropol-S) & $72 / 100 \%$ \\
\hline
\end{tabular}




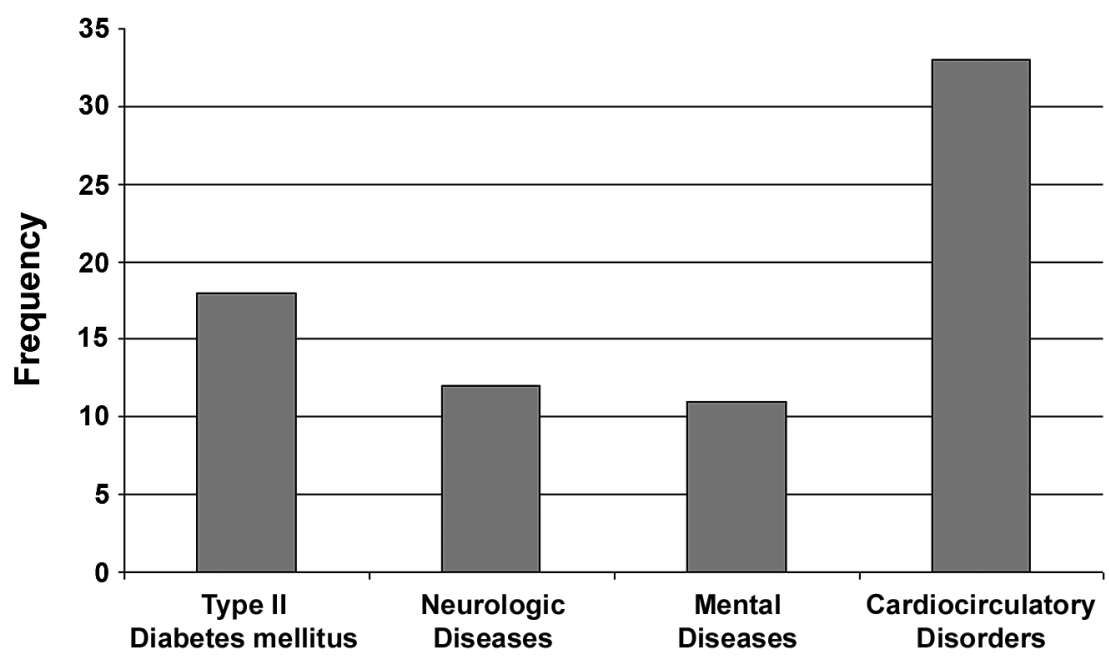

Figure 1 - Concomitant diseases. There was no significant association between concomitant diseases and outcome of treatment.

\section{Urodynamic Testing}

The average bladder capacity was $185 \mathrm{~mL}$ ( \pm $32 \mathrm{~mL}$ ) and patients had an average voiding frequency of $16( \pm 3)$ times a day. In 33 patients, bladder capacity was significantly improved by $110 \mathrm{~mL}( \pm 25 \mathrm{~mL})(\mathrm{p}=$ $0.0001)$. Voiding frequency decreased significantly to $7( \pm 2)$ times a day $(p=0.003)$. Nineteen patients $(26$ $\%$ ) had detrusor overactivity, which was terminal in

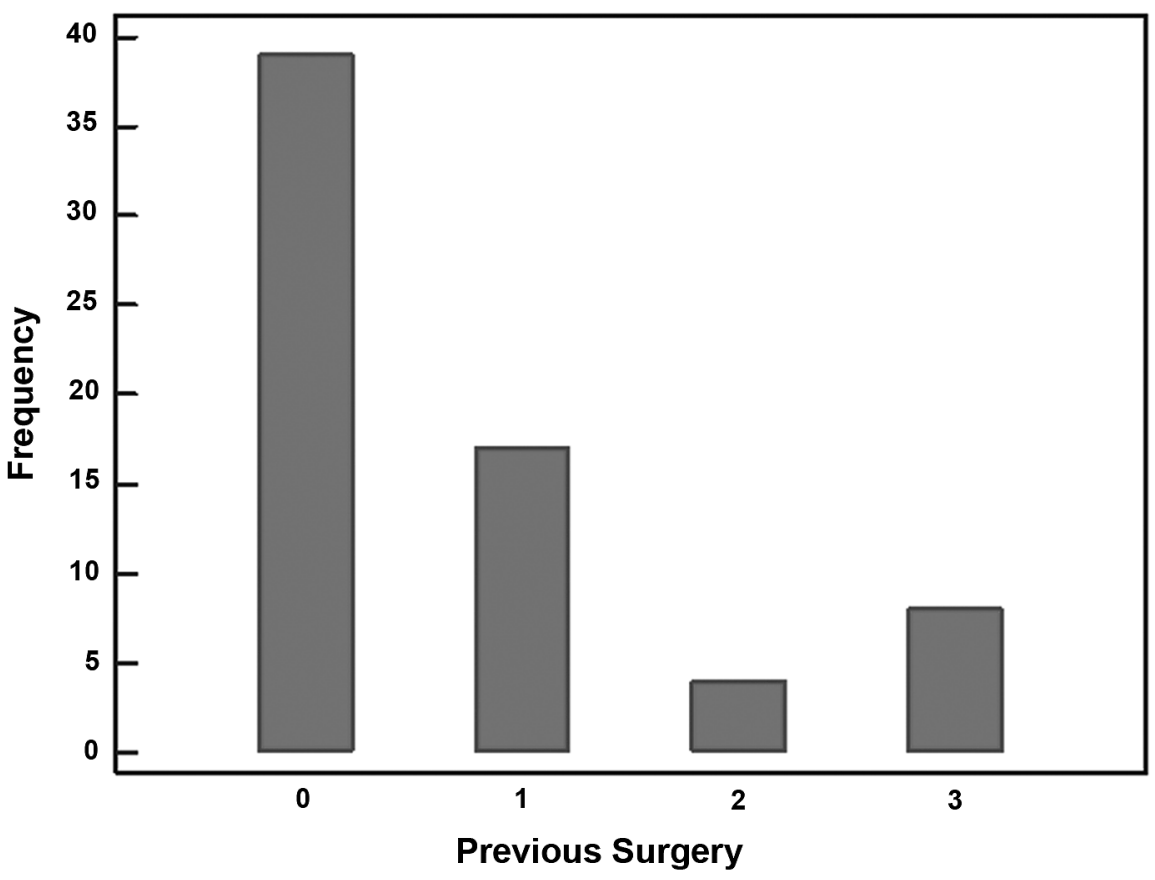

Figure 2 - Previous surgery. $0=$ none; $1=$ TVT; 2 = botox injection; 3 = prolapse surgery. 
15 of them $(21 \%)$ and phasic in the other 4 patients $(30.8 \%)$. In $7(37 \%)$ of the $19(26 \%)$ patients, it was not possible to evaluate involuntary detrusor contraction following treatment. The first urge was premature in $41(57 \%)$ patients before treatment versus $18(25 \%)$ after treatment (Tables-2 and 3).

No abnormal residual urine volumes were identified either sonographically or clinically.

\section{Nocturia, Quality of Life, and Sexuality}

Nocturia showed a tendency to improve from $5( \pm 2)$ to $2( \pm 1)$.

Quality of life was improved in 54 patients $(75 \%)$; patients $(\mathrm{p}=0.002)$. Thirty-nine $(54 \%)$ patients had improved sexuality $(\mathrm{p}=0.001)$ in terms of sexual sensations and sexual satisfaction. Quality of life was unchanged in 15 (21\%) and worsened in three (4\%), Figure-3.

\section{Adverse Effects}

Twelve of the women (17\%) developed reactive hypertension during treatment, which returned to normal without intervention.

Signs of dysuria and hematuria were observed in a total of 21 patients $(29 \%)$. Urinary tract infection occurred in 10 women (14\%) and was treated with a 7-day regimen of $250 \mathrm{mg}$ ciprofloxacin ( 2 x 1 tablet), followed by a urine test. One patient developed urinary retention, which disappeared after she was catheterized twice.

\section{COMMENTS}

Although the number of patients investigated in our study is still too small to draw any general conclusions, our data clearly suggests that the symptoms of overactive bladder tend to markedly improve using electromotive drug administration (EMDA) for treatment. Our therapeutic approach cannot be compared with other currently available treatments using agents such as oxybutynin, protamine sulfate, and dimethyl sulfoxide $(9,10)$ because these procedures use very different modes of administration.

The combination of drugs we used was specifically selected to control urge and pain as well as chronic inflammation of bladder tissue, which is present in most affected patients. The approach used here allows simultaneous bladder distension for improving bladder capacity without the need for anesthesia. We attribute the improvement observed in our patients to the drugs administered by iontophoresis (EMDA) and bladder distension since they did not receive additional anticholinergic treatment or physical therapy. From a strictly scientific point of view, our study population should have been compared with a con-

Table 2 - Results of urodynamic testing. There was significant improvement in the urodynamic parameters investigated.

\begin{tabular}{lcc}
\hline & Before & After \\
\hline Premature first urge & 41 & 18 \\
Detrusor contraction & 19 & 7 \\
Bladder capacity $(\mathrm{mL})$ & $185 \pm 32$ & $110 \pm 25$ \\
\hline
\end{tabular}

Table 3 - Comparison of group A (sensory urge) and group B (detrusor overactivity) The only significant difference in post-therapeutic improvement was found between the two groups for voiding frequency.

\begin{tabular}{lcccc}
\hline & $\begin{array}{c}\text { Bladder } \\
\text { Capacity }\end{array}$ & $\begin{array}{c}\text { Voiding Frequency } \\
\text { (per day) }\end{array}$ & $\begin{array}{c}\text { N of Involuntary } \\
\text { Loss of Urine }\end{array}$ & $\begin{array}{c}\text { Involuntary Loss } \\
\text { of Urine } \\
{[<5 \text { pro Tag }]}\end{array}$ \\
\hline (A) Sensory urge (41) & $281 \mathrm{~mL}$ & 9 & 18 & 23 \\
(B) Detrusor overactivity (19) & $264 \mathrm{~mL}$ & 5 & 11 & 8 \\
\hline
\end{tabular}




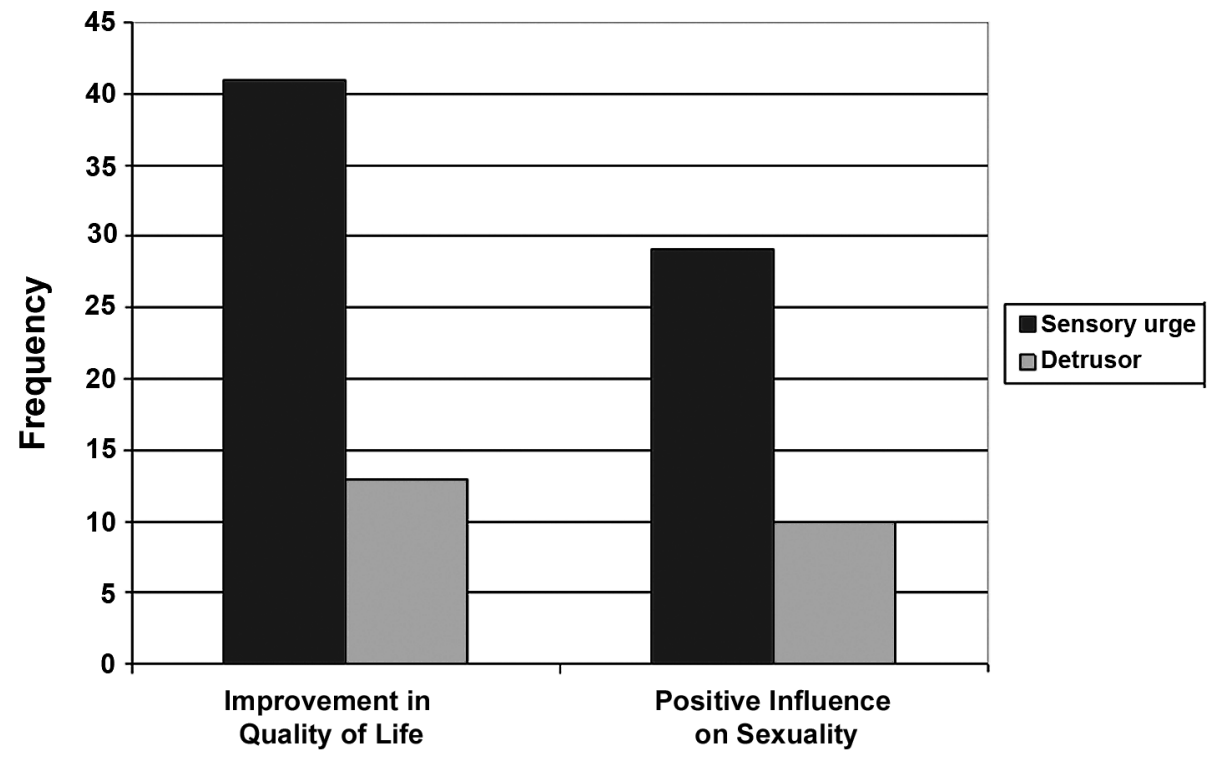

Figure 3 - Post-therapeutic quality of life and sexuality in group A (sensory urge) and group B (detrusor overactivity). Significant improvement in quality of life was observed.

trol group receiving placebo. Ethical considerations precluded this approach because most of our patients had very long histories of chronic disease. Investigation of a larger group of patients might provide sufficient evidence for the effectiveness of EMDA. Both above-mentioned effects of this therapeutic approach were observed in our patients: voiding frequency was reduced in our patients while bladder capacity was markedly increased. These effects in turn improved quality of life in general as well as sexuality (less involuntary urine loss during and around intercourse, greater enjoyment of sexual sensations, more frequent orgasms). We also found that an abnormally high BMI tended to impair pelvic floor function, suggesting that affected patients should be helped to lose weight by means of dietary therapy adapted for this population. Analysis of the medications and physiotherapeutic approaches used in the patients before EMDA treatment revealed no association with outcome. However, a slightly improved response rate was seen in the patients who were treated with oxybutynin transdermal patches (kentera $\left.{ }^{\circledR}\right)$ but the difference was not significant. The adverse effects seen in our patients were moderate and comparable to those reported in the literature (11). However, the circulatory effects associated with this kind of treatment suggest that closer cardiologic workup should be performed prior to treatment. The current pre-therapeutic diagnostic workup has already been supplemented by an ECG and a medical consultation. Circulatory monitoring is needed in all cases. Quality of life was unchanged in $15(21 \%)$ and worsened in three (4\%).

Three patients (4\%) who did not report improvement had poor compliance in that the treatment sessions were performed at very irregular intervals. We assume that this reduced the efficiency of EMDA but have no definitive evidence because the time differences were too small ( \pm 3 days).

$\mathrm{OAB}$ is not a normal condition in adults regardless of age, sex, and degree of mobility. While not life-threatening, the condition is associated with an unnecessary economic burden and may lead to isolation, depression, embarrassment, and low selfesteem of affected individuals. The results presented here suggest that very good outcome can be achieved with EMDA, which allows local drug administration without severe adverse effects. Our results deserve to be further corroborated in larger studies inves- 
tigating EMDA as second-line treatment option in patients with overactive bladder, since we observed an improved quality of life during one-year follow-up. Therefore, we think it is very promising to continue treatment, assess intermediate-term results, and adjust the therapeutic approach as needed.

\section{CONFLICT OF INTEREST}

None declared.

\section{REFERENCES}

1. Schumacher S: Epidemiology and pathophysiology of overactive bladder. Urologe A. 2006; 45: 822-5.

2. Hampel C, Gillitzer R, Pahernik S, Hohenfellner M, Thüroff JW: Epidemiology and etiology of overactive bladder. Urologe A. 2003; 42: 776-86.

3. Bump RC, Mattiasson A, Bø K, Brubaker LP, DeLancey JO, Klarskov P, et al.: The standardization of terminology of female pelvic organ prolapse and pelvic floor dysfunction. Am J Obstet Gynecol. 1996; 175: 10-7.

4. Roy S, Caillouette JC, Roy T, Faden JS: Vaginal pH is similar to follicle-stimulating hormone for menopause diagnosis. Am J Obstet Gynecol. 2004; 190: 1272-7.
5. Tunn R, Schaer G, Peschers U, Bader W, Gauruder A, Hanzal E, et al.: Updated recommendations on ultrasonography in urogynecology. Int Urogynecol J Pelvic Floor Dysfunct. 2005; 16: 236-41.

6. Reese PR, Pleil AM, Okano GJ, Kelleher CJ: Multinational study of reliability and validity of the King's Health Questionnaire in patients with overactive bladder. Qual Life Res. 2003; 12: 427-42.

7. Ahlers CJ, Schaefer GA, Beier KM: Fragebogen zum sexuellen Erleben und Verhalten. Sexuologie 2004; 11: 74-97.

8. Schüssler B, Laycock J, Norton P, Stanton S: Pelvicfloor reeducation: principles and practice. In: Laycock J: (ed.), Clinical Evaluation of the Pelvic Floor. Springer Verlag, London. 1994; pp.42-48.

9. Giannantoni A, Di Stasi SM, Chancellor MB, Costantini E, Porena M: New frontiers in intravesical therapies and drug delivery. Eur Urol. 2006; 50: 1183-93; discussion 1193.

10. Di Stasi SM, Giannantoni A, Vespasiani G, Navarra P, Capelli G, Massoud R, et al.: Intravesical electromotive administration of oxybutynin in patients with detrusor hyperreflexia unresponsive to standard anticholinergic regimens. J Urol. 2001; 165: 491-8.

11. Hinkel A, Pannek J: Transient ischemic attack after electromotive drug administration for chronic non-infectious cystitis: report of two similar cases. Neurourol Urodyn. 2004; 23: 180-2.

\section{Correspondence address:}

Dr. Annett Gauruder-Burmester

Deutsches Beckenbodenzentrum

Grobe Hamburger Str. 5-11

Berlin, 10115, Germany

Fax: + 030 2311-2728

E-mail: a.gauruder@googlemail.com 